\title{
Set-Valued Means
}

\section{Kazimierz Nikodem ${ }^{1}$}

Received: 15 July 2019 / Accepted: 16 January 2020 / Published online: 3 February 2020

(C) The Author(s) 2020

\section{Abstract}

The notion of set-valued means is introduced. Set-valued counterparts of the arithmetic, quasi-arithmetic and Lagrangian means are investigated and various properties of them are presented.

Keywords Means $\cdot$ Set-valued maps $\cdot$ Set-valued means $\cdot$ Inequalities between means

Mathematics Subject Classification (2010) Primary 26E60; Secondary 26B25 · 26E25 . 39B62

\section{Introduction}

Let $I \subset \mathbb{R}$ be an interval and $n \geq 2$ be an integer. A function $M: I^{n} \rightarrow I$ is said to be a mean if

$$
\min \left\{x_{1}, \ldots, x_{n}\right\} \leq M\left(x_{1}, \ldots, x_{n}\right) \leq \max \left\{x_{1}, \ldots, x_{n}\right\}
$$

for all $x_{1}, \ldots, x_{n} \in I$.

This formal definition was probably used first time by Cauchy [5] almost two hundred years ago, but the general idea of means is much older. The classical means, such as arithmetic, geometric or harmonic, were known already in the ancient time. At present the theory of means is an extensive mathematical theory having various applications in mathematics itself as well as in economics, information theory, engineering and natural science. There are books and numerous papers devoted to it (see e.g. $[1,3,4,6-10,12]$ and the references given there).

The aim of this paper is to generalize the classical definition of mean (1) to the case of setvalued means in vector spaces. We extend the notion of arithmetic mean defining a family of set-valued means containing the arithmetic one and present various properties of them. Set-valued counterparts of the quasi-arithmetic and Lagrangian means are also investigated.

Kazimierz Nikodem

knikodem@ath.bielsko.pl

1 Department of Mathematics, University of Bielsko-Biała, ul. Willowa 2, 43-309,

Bielsko-Biała, Poland 
Let $X$ be a real vector space and $D$ be a convex nonempty subset of $X$. Denote by $S(D)$ the family of all nonempty subsets of $D$. We say that a function $M: D^{n} \rightarrow S(D)$ is a set-valued mean if

$$
M\left(x_{1}, \ldots, x_{n}\right) \subset \operatorname{conv}\left\{x_{1}, \ldots, x_{n}\right\},
$$

for all $x_{1}, \ldots, x_{n} \in D$.

Clearly, if $X=\mathbb{R}, D=I$ and $M$ is single-valued, then condition (2) reduces to (1). Note also that any $M: D^{n} \rightarrow S(D)$ defined by (2) is reflexive, that is $M(x, \ldots, x)=\{x\}$ for every $x \in D$.

A set-valued mean $M: D^{n} \rightarrow S(D)$ is said to be symmetric, if

$$
M\left(x_{\sigma(1)}, \ldots, x_{\sigma(n)}\right)=M\left(x_{1}, \ldots, x_{n}\right)
$$

for all $x_{1}, \ldots, x_{n} \in D$ and every permutation $\sigma:\{1, \ldots, n\} \rightarrow\{1, \ldots, n\}$.

$M$ is homogenous if

$$
M\left(s x_{1}, \ldots, s x_{n}\right)=s M\left(x_{1}, \ldots, x_{n}\right)
$$

for all $x_{1}, \ldots, x_{n} \in D$ and every $s \in \mathbb{R}$ such that $s x_{i} \in D, i=1, \ldots, n$.

We say that $M$ is concave if

$$
M(\lambda x+(1-\lambda) y) \subset \lambda M(x)+(1-\lambda) M(y),
$$

for all $x=\left(x_{1}, \ldots, x_{n}\right), y=\left(y_{1}, \ldots, y_{n}\right) \in D^{n}$ and $\lambda \in[0,1] . M$ is called convex if the opposite inclusions hold.

\section{Set-valued extension of the arithmetic mean}

Let us start with an example in which we define a family of set-valued means extending the classical arithmetic mean. For a given $x=\left(x_{1}, \ldots, x_{n}\right) \in D^{n}$ we put $\bar{x}:=\frac{1}{n}\left(x_{1}+\cdots+x_{n}\right)$.

Example 1 Let $M_{0}, M_{1}: D^{n} \rightarrow S(D)$ be defined by

$$
\begin{gathered}
M_{0}\left(x_{1}, \ldots, x_{n}\right)=\bar{x}, x_{1}, \ldots, x_{n} \in D \\
M_{1}\left(x_{1}, \ldots, x_{n}\right)=\operatorname{conv}\left\{x_{1}, \ldots, x_{n}\right\}, x_{1}, \ldots, x_{n} \in D .
\end{gathered}
$$

More generally, for every $t \in[0,1]$ define $M_{t}: D^{n} \rightarrow S(D)$ by

$$
M_{t}\left(x_{1}, \ldots, x_{n}\right)=t \operatorname{conv}\left\{x_{1}, \ldots, x_{n}\right\}+(1-t) \bar{x}, x_{1}, \ldots, x_{n} \in D .
$$

It is clear that $M_{t}$ are set-valued means and

$$
M_{0}\left(x_{1}, \ldots, x_{n}\right) \subset M_{t}\left(x_{1}, \ldots, x_{n}\right) \subset M_{1}\left(x_{1}, \ldots, x_{n}\right),
$$

for every $t \in[0,1]$.

Theorem 1 Let $D$ be a convex subset of a real vector space $X$ and let $t \in[0,1]$. The setvalued means $M_{t}: D^{n} \rightarrow S(D)$ defined by (3) are symmetric, homogenous and concave. Moreover, they have the following comparison property:

$$
0 \leq s \leq t \leq 1 \Rightarrow M_{s}\left(x_{1}, \ldots, x_{n}\right) \subset M_{t}\left(x_{1}, \ldots, x_{n}\right),
$$

for all $x_{1}, \ldots, x_{n} \in D$. 
Proof The symmetry and homogeneity of $M_{t}$ are clear. To prove the concavity, fix $x=$ $\left(x_{1}, \ldots, x_{n}\right), y=\left(y_{1}, \ldots, y_{n}\right) \in D^{n}, \lambda \in[0,1]$, and take a point

$$
\begin{aligned}
z & \in M_{t}(\lambda x+(1-\lambda) y) \\
& =t \operatorname{conv}\left\{\lambda x_{1}+(1-\lambda) y_{1}, \ldots, \lambda x_{n}+(1-\lambda) y_{n}\right\}+(1-t) \overline{(\lambda x+(1-\lambda) y)} .
\end{aligned}
$$

Then

$$
z=t z^{\prime}+(1-t) \overline{(\lambda x+(1-\lambda) y)},
$$

where $z^{\prime} \in \operatorname{conv}\left\{\lambda x_{1}+(1-\lambda) y_{1}, \ldots, \lambda x_{n}+(1-\lambda) y_{n}\right\}$. By the definition of convex hull, there exist $\alpha_{1}, \ldots, \alpha_{n} \geq 0$ with $\alpha_{1}+\cdots+\alpha_{n}=1$ such that

$$
\begin{aligned}
z^{\prime} & =\alpha_{1}\left(\lambda x_{1}+(1-\lambda) y_{1}\right)+\cdots+\alpha_{n}\left(\lambda x_{n}+(1-\lambda) y_{n}\right) \\
& =\lambda\left(\alpha_{1} x_{1}+\cdots+\alpha_{n} x_{n}\right)+(1-\lambda)\left(\alpha_{1} y_{1}+\cdots+\alpha_{n} y_{n}\right) \\
& \in \lambda \operatorname{conv}\left\{x_{1}, \ldots, x_{n}\right\}+(1-\lambda) \operatorname{conv}\left\{y_{1}, \ldots, y_{n}\right\} .
\end{aligned}
$$

We have also

$$
\overline{(\lambda x+(1-\lambda) y)}=\lambda \bar{x}+(1-\lambda) \bar{y} .
$$

By (4), (5) and (6), we obtain

$$
\begin{aligned}
z & \in t\left(\lambda \operatorname{conv}\left\{x_{1}, \ldots, x_{n}\right\}+(1-\lambda) \operatorname{conv}\left\{y_{1}, \ldots, y_{n}\right\}+(1-t)(\lambda \bar{x}+(1-\lambda) \bar{y})\right. \\
& =\lambda\left(t \operatorname{conv}\left\{x_{1}, \ldots, x_{n}\right\}+(1-t) \bar{x}\right)+(1-\lambda)\left(t \operatorname{conv}\left\{y_{1}, \ldots, y_{n}\right\}+(1-t) \bar{y}\right) \\
& =\lambda M_{t}(x)+(1-\lambda) M_{t}(y),
\end{aligned}
$$

which proves that $M_{t}$ is concave.

To prove the comparison property fix $0 \leq s<t \leq 1$ and take any $z \in M_{s}\left(x_{1}, \ldots, x_{n}\right)$. There exist $\alpha_{1}, \ldots, \alpha_{n} \geq 0$ with $\alpha_{1}+\cdots+\alpha_{n}=1$ such that

$$
z=\sum_{i=1}^{n} \alpha_{i} x_{i}+(1-s) \bar{x}=t \sum_{i=1}^{n} \frac{s}{t} \alpha_{i} x_{i}+(1-t) \bar{x}+(t-s) \bar{x} .
$$

Since

$$
t \sum_{i=1}^{n} \frac{s}{t} \alpha_{i} x_{i}+(t-s) \bar{x}=t \sum_{i=1}^{n}\left(\frac{s}{t} \alpha_{i}+\frac{t-s}{n t}\right) x_{i} \in t \operatorname{conv}\left\{x_{1}, \ldots, x_{n}\right\},
$$

by (7) we get $x \in M_{t}\left(x_{1}, \ldots, x_{n}\right)$. This finishes the proof.

Remark 2 The mean $M_{0}$ is single-valued and it is an affine function from $D^{n}$ to $D$. Since $M_{0}\left(x_{1}, \ldots, x_{n}\right) \in M_{t}\left(x_{1}, \ldots, x_{n}\right),\left(x_{1}, \ldots, x_{n}\right) \in D^{n}$, this shows that every set-valued mean $M_{t}, t \in(0,1]$, has an affine selection.

For arbitrary $t \in(0,1]$ the set-valued means $M_{t}$ need not be convex. For example, assume that $D=[0,1], n=2$ and take $x=(1,0), y=(0,1)$. Then for the set-valued mean $M_{t}:[0,1]^{2} \rightarrow[0,1]$ we have

$$
M_{t}(x)=\left[\frac{1}{2}-\frac{t}{2}, \frac{1}{2}+\frac{t}{2}\right]=M_{t}(y) \text { and } M_{t}\left(\frac{x+y}{2}\right)=\left\{\frac{1}{2}\right\} .
$$

Therefore, for each $t \in(0,1]$,

$$
\frac{1}{2} M_{t}(x)+\frac{1}{2} M_{t}(y)=\left[\frac{1}{2}-\frac{t}{2}, \frac{1}{2}+\frac{t}{2}\right] \nsubseteq M_{t}\left(\frac{x+y}{2}\right),
$$

which shows that $M_{t}$ is not convex. 
Assume now that $X, Y$ are topological vector spaces and $D \subset X$. A set-valued map $F: D \rightarrow S(Y)$ is said to be continuous at a point $x_{0} \in D$ if for every neighbourhood $W$ of zero in $Y$ there exists a neighbourhood $U$ of zero in $X$ such that

$$
F\left(x_{0}\right) \subset F(x)+W \text { and } F(x) \subset F\left(x_{0}\right)+W
$$

for every $x \in\left(x_{0}+U\right) \cap D$. If only the first (the second) inclusions are fulfilled, $F$ is called lower semicontinuous (upper semicontinuous) at $x_{0}$. Lower (resp. upper) semicontinuity as we have defined is a stronger (resp. weaker) requirement than the usage of this terminology by Kuratowski.

The next result describes continuity properties of the set-valued means $M_{t}$.

Theorem 3 Let $X$ be a Hausdorff topological vector space and $D \subset X$ be a convex set. For every $t \in[0,1]$ the set-valued mean $M_{t}: D^{n} \rightarrow S(D)$ is continuous on $D^{n}$. Moreover, for every fixed $\left(x_{1}, \ldots, x_{n}\right) \in X^{n}$ the map $t \mapsto M_{t}\left(x_{1}, \ldots, x_{n}\right)$ is continuous on $[0,1]$.

Proof Since the function $\left(x_{1}, \ldots, x_{n}\right) \mapsto \frac{1}{n}\left(x_{1}+\cdots+x_{n}\right)$ is continuous, it suffices to show that the set-valued map conv : $\left(x_{1}, \ldots, x_{n}\right) \mapsto \operatorname{conv}\left\{x_{1}, \ldots, x_{n}\right\}$ is continuous. To this aim fix $\left(x_{1}^{0}, \ldots, x_{n}^{0}\right) \in D^{n}$ and take a neighbourhood $W$ of zero in $X$. There exist a balanced neighbourhood $V$ of zero in $X$ such that the algebraic sum of $n$ copies of $V$ $V+\cdots+V \subset W$. Let

$$
\left(x_{1}, \ldots, x_{n}\right) \in\left(\left(x_{1}^{0}, \ldots, x_{n}^{0}\right)+V^{n}\right) \cap D^{n},
$$

that is $x_{i} \in\left(x_{i}^{0}+V\right) \cap D, i=1, \ldots, n$. Then, for all $\alpha_{1}, \ldots, \alpha_{n} \geq 0$ with $\alpha_{1}+\cdots+\alpha_{n}=1$ we have

$$
\sum_{i=1}^{n} \alpha_{i} x_{i} \in \sum_{i=1}^{n} \alpha_{i}\left(x_{i}+V\right) \subset \sum_{i=1}^{n} \alpha_{i} x_{i}^{0}+(V+\cdots+V) \subset \operatorname{conv}\left\{x_{1}^{0}, \ldots, x_{n}^{0}\right\}+W,
$$

and hence

$$
\operatorname{conv}\left\{x_{1}, \ldots, x_{n}\right\} \subset \operatorname{conv}\left\{x_{1}^{0}, \ldots, x_{n}^{0}\right\}+W .
$$

This means that the map conv is upper semicontinuous at $\left(x_{1}^{0}, \ldots, x_{n}^{0}\right)$.

Since $V$ is balanced and $\left(x_{1}^{0}, \ldots, x_{n}^{0}\right) \in D^{n}$, by (8) we have also

$$
\left(x_{1}^{0}, \ldots, x_{n}^{0}\right) \in\left(\left(x_{1}, \ldots, x_{n}\right)+V^{n}\right) \cap D^{n},
$$

and hence, analogously as above,

$$
\operatorname{conv}\left\{x_{1}^{0}, \ldots, x_{n}^{0}\right\} \subset \operatorname{conv}\left\{x_{1}, \ldots, x_{n}\right\}+W .
$$

This shows that the map conv is lower semicontinuous at $\left(x_{1}^{0}, \ldots, x_{n}^{0}\right)$ and finishes the proof of the continuity of $M_{t}$.

The second statement follows from the facts that for every fixed $\left(x_{1}, \ldots, x_{n}\right) \in X^{n}$ the set $\operatorname{conv}\left\{x_{1}, \ldots, x_{n}\right\}$ is bounded and for any bounded set $B \subset X$ the map $t \mapsto t B$ is continuous on $\mathbb{R}$ (see e.g. [11, Lemma 3.2]). 


\section{Quasi-arithmetic-type set-valued means}

Let $I, J \subset \mathbb{R}$ be intervals. If a function $f: I \rightarrow J$ is continuous and strictly monotonic, then $A_{f}: I^{n} \rightarrow I$ defined by

$$
A_{f}\left(x_{1}, \ldots, x_{n}\right)=f^{-1}\left(\frac{f\left(x_{1}\right)+\cdots+f\left(x_{n}\right)}{n}\right)
$$

is the quasi-arithmetic mean generated by $f$ (see e.g. [8] and the references therein). In a similar way we can define its set-valued counterpart putting, for a given set-valued map $F: I \rightarrow S(J)$,

$$
A_{F}\left(x_{1}, \ldots, x_{n}\right)=F^{+}\left(\frac{F\left(x_{1}\right)+\cdots+F\left(x_{n}\right)}{n}\right),
$$

where $F^{+}(B):=\{x \in I: F(x) \subset B\}$. However, without any additional assumptions, $A_{F}$ need not be a set-valued mean.

Example 2 Let $n=2$ and $F:[0,1] \rightarrow S([0,1])$ be defined by $F(x)=\left[x^{2}, \sqrt{x}\right], x \in$ $[0,1]$. Then

$$
A_{F}(0,1)=F^{+}\left(\frac{F(0)+F(1)}{2}\right)=F^{+}\left(\left\{\frac{1}{2}\right\}\right)=\emptyset .
$$

Since, by the definition, values of set-valued means are nonempty, this shows that $A_{F}$ is not a mean.

Example 3 Let $n=2$ and $F: \mathbb{R} \rightarrow S(\mathbb{R})$ be defined by $F(x)=[x,+\infty), x \in \mathbb{R}$. Then for any $x, y \in \mathbb{R}$

$$
A_{F}(x, y)=F^{+}\left(\frac{F(x)+F(y)}{2}\right)=\left[\frac{x+y}{2},+\infty\right) \nsubseteq \operatorname{conv}\{x, y\} .
$$

Thus $A_{F}$ is not a mean.

The next theorem gives some conditions under which $A_{F}$ is a set-valued mean.

Theorem 4 Let $f, g: I \rightarrow J$ be strictly increasing functions such that $f$ is concave and $g$ is convex. Assume that $f \leq g$ on I and $F(x)=[f(x), g(x)]$ for all $x \in I$. Then, for every $n \geq 2$, the map $A_{F}: I^{n} \rightarrow S(I)$ given by

$$
A_{F}\left(x_{1}, \ldots, x_{n}\right)=F^{+}\left(\frac{1}{n} \sum_{i=1}^{n} F\left(x_{i}\right)\right), x_{1}, \ldots, x_{n} \in I
$$

is a set-valued mean.

Proof Fix $n \geq 2$ and take $x_{1}, \ldots, x_{n} \in I$. We first prove that $A_{F}\left(x_{1}, \ldots, x_{n}\right) \neq \varnothing$. By the concavity of $f$ and convexity of $g$, we have

$$
f\left(\frac{1}{n} \sum_{i=1}^{n} x_{i}\right) \geq \frac{1}{n} \sum_{i=1}^{n} f\left(x_{i}\right) \text { and } g\left(\frac{1}{n} \sum_{i=1}^{n} x_{i}\right) \leq \frac{1}{n} \sum_{i=1}^{n} g\left(x_{i}\right),
$$


and hence

$$
\begin{aligned}
F\left(\frac{1}{n} \sum_{i=1}^{n} x_{i}\right) & =\left[f\left(\frac{1}{n} \sum_{i=1}^{n} x_{i}\right), g\left(\frac{1}{n} \sum_{i=1}^{n} x_{i}\right)\right] \\
& \subset\left[\frac{1}{n} \sum_{i=1}^{n} f\left(x_{i}\right), \frac{1}{n} \sum_{i=1}^{n} g\left(x_{i}\right)\right]=\frac{1}{n} \sum_{i=1}^{n} F\left(x_{i}\right) .
\end{aligned}
$$

Consequently,

$$
\frac{1}{n} \sum_{i=1}^{n} x_{i} \in F^{+}\left(\frac{1}{n} \sum_{i=1}^{n} F\left(x_{i}\right)\right)=A_{F}\left(x_{1}, \ldots, x_{n}\right),
$$

which proves that $A_{F}\left(x_{1}, \ldots, x_{n}\right) \neq \emptyset$.

Now, fix an $x \in A_{F}\left(x_{1}, \ldots, x_{n}\right)$. Then

$$
F(x) \subset \frac{1}{n} \sum_{i=1}^{n} F\left(x_{i}\right)=\left[\frac{1}{n} \sum_{i=1}^{n} f\left(x_{i}\right), \frac{1}{n} \sum_{i=1}^{n} g\left(x_{i}\right)\right],
$$

which implies that

$$
f(x) \geq \frac{1}{n} \sum_{i=1}^{n} f\left(x_{i}\right) \text { and } g(x) \leq \frac{1}{n} \sum_{i=1}^{n} g\left(x_{i}\right) .
$$

Since $f$ and $g$ are strictly increasing, from (10) we get

$$
x \geq \min \left\{x_{1}, \ldots, x_{n}\right\} \text { and } x \leq \max \left\{x_{1}, \ldots, x_{n}\right\} .
$$

Hence

$$
A_{F}\left(x_{1}, \ldots, x_{n}\right) \subset\left[\min \left\{x_{1}, \ldots, x_{n}\right\}, \max \left\{x_{1}, \ldots, x_{n}\right\}\right]=\operatorname{conv}\left\{x_{1}, \ldots, x_{n}\right\},
$$

which finishes the proof.

Remark 5 The assumptions that $f$ is concave, $g$ is convex and $f \leq g$ imply that the setvalued map $F(x)=[f(x), g(x)]$ is concave. If we have any concave set-valued map $F^{\prime}$ : $I \rightarrow S(I)$, then the set-valued mean $A_{F^{\prime}}$ generated by $F^{\prime}$ has nonempty values (because $\left.\frac{1}{n}\left(x_{1}+\cdots+x_{n}\right) \in A_{F^{\prime}}\left(x_{1}, \ldots, x_{n}\right)\right)$, but $A_{F^{\prime}}\left(x_{1}, \ldots, x_{n}\right) \nsubseteq \operatorname{conv}\left\{x_{1}, \ldots, x_{n}\right\}$, in general. Consider, for example, the set-valued map $F^{\prime}: \mathbb{R} \rightarrow S(\mathbb{R})$ defined by $F^{\prime}(x)=[0,|x|], x \in$ $\mathbb{R}$. Then, for $n=2$, we have

$$
A_{F^{\prime}}(-1,2)=F^{\prime+}\left(\frac{[0,1]+[0,3]}{2}\right)=F^{\prime+}([0,2])=[-2,2] \nsubseteq \operatorname{conv}\{-1,2\} \text {. }
$$

Assume now that $M, N: I^{n} \rightarrow I$ are two means such that $M \leq N$ on $I$. Then the map $M N: I^{n} \rightarrow S(I)$ defined by

$$
M N\left(x_{1}, \ldots, x_{n}\right)=\left[M\left(x_{1}, \ldots, x_{n}\right), N\left(x_{1}, \ldots, x_{n}\right)\right], x_{1}, \ldots, x_{n} \in I,
$$

is a set-valued mean. Using this observation we can construct various set-valued means from single-valued ones. For instance, consider the classical harmonic, geometric, arithmetic and quadratic means defined for $x_{1}, \ldots, x_{n}>0$ by

$$
\begin{gathered}
H\left(x_{1}, \ldots, x_{n}\right)=\frac{n}{\frac{1}{x_{1}}+\cdots+\frac{1}{x_{n}}}, \quad G\left(x_{1}, \ldots, x_{n}\right)=\sqrt[n]{x_{1} \cdots x_{n}}, \\
A\left(x_{1}, \ldots, x_{n}\right)=\frac{x_{1}+\cdots+x_{n}}{n}, \quad K\left(x_{1}, \ldots, x_{n}\right)=\sqrt{\frac{x_{1}^{2}+\cdots+x_{n}^{2}}{n}} .
\end{gathered}
$$


All these means are quasi-arithmetic means generated by the functions $f(x)=-1 / x$, $f(x)=\ln x, f(x)=x$ and $f(x)=x^{2}$, respectively. It is known that

$$
H\left(x_{1}, \ldots, x_{n}\right) \leq G\left(x_{1}, \ldots, x_{n}\right) \leq A\left(x_{1}, \ldots, x_{n}\right) \leq K\left(x_{1}, \ldots, x_{n}\right),
$$

for all $x_{1}, \ldots, x_{n}>0$. Using these inequalities we can define the following set valued means:

$$
\begin{array}{rll}
H G(x):=[H(x), G(x)], & H A(x):=[H(x), A(x)], & H K(x):=[H(x), K(x)], \\
G A(x):=[G(x), A(x)], & G K(x):=[G(x), K(x)], & A K(x):=[A(x), K(x)],
\end{array}
$$

where $x=\left(x_{1}, \ldots, x_{n}\right) \in(0, \infty)^{n}$.

Observe that all but the first of the above set-valued means are examples of the means (9) obtained for appropriate generators $F$.

Example 4 Let $F_{1}(x)=\left[\ln x, x^{2}\right], x>0$. Then $F_{1}$ satisfies the assumptions of Theorem 4 and therefore $A_{F_{1}}$ is a set-valued mean (in particular, it has nonempty values). Assume, for simplicity, that $n=2$. Since

$$
A_{F_{1}}(x, y)=F_{1}^{+}\left(\frac{1}{2}\left(\left[\ln x, x^{2}\right]+\left[\ln y, y^{2}\right]\right)\right)=F_{1}^{+}\left(\left[\ln \sqrt{x y}, \frac{x^{2}+y^{2}}{2}\right]\right),
$$

we obtain

$$
z \in A_{F_{1}}(x, y) \Leftrightarrow\left[\ln z, z^{2}\right] \subset\left[\ln \sqrt{x y}, \frac{x^{2}+y^{2}}{2}\right] \Leftrightarrow z \in\left[\sqrt{x y}, \sqrt{\frac{x^{2}+y^{2}}{2}}\right] .
$$

This proves that $A_{F_{1}}(x, y)=G K(x, y)$ for all $x, y>0$.

Now, let $F_{2}(x)=\left[-\frac{1}{x}, x\right], x>0$. Then

$$
A_{F_{2}}(x, y)=F_{2}^{+}\left(\frac{1}{2}\left[-\frac{1}{x}, x\right]+\frac{1}{2}\left[-\frac{1}{y}, y\right]\right)=F_{2}^{+}\left(\left[\frac{x+y}{-2 x y}, \frac{x+y}{2}\right]\right) .
$$

Hence

$$
z \in A_{F_{2}}(x, y) \Leftrightarrow\left[-\frac{1}{z}, z\right] \subset\left[\frac{x+y}{-2 x y}, \frac{x+y}{2}\right] \Leftrightarrow z \in\left[\frac{2 x y}{x+y}, \frac{x+y}{2}\right],
$$

which shows that $A_{F_{2}}(x, y)=H A(x, y)$ for all $x, y>0$.

Similarly, taking one by one $F_{3}(x)=[\ln x, x], F_{4}(x)=\left[x, x^{2}+1\right]$ and $F_{5}(x)=$ $\left[-\frac{1}{x}, x^{2}\right]$ we can show that $A_{F_{3}}(x, y)=G A(x, y), A_{F_{4}}(x, y)=A K(x, y)$ and $A_{F_{5}}(x, y)=H K(x, y)$, for all $x, y>0$.

Observe also that some of the inequalities (11) between the classical means can be obtained as corollaries to Theorem 4 . Let us formulate explicitly a more general result.

Theorem 6 Let $f, g: I \rightarrow J$ be strictly increasing functions such that $f$ is concave and $g$ is convex. Then

$$
A_{f}\left(x_{1}, \ldots, x_{n}\right) \leq A\left(x_{1}, \ldots, x_{n}\right) \leq A_{g}\left(x_{1}, \ldots, x_{n}\right)
$$

for all $x_{1}, \ldots, x_{n} \in I$.

Proof Fix $x_{1}, \ldots, x_{n} \in I$. By the concavity of $f$ and convexity of $g$ we have

$$
f\left(\frac{1}{n} \sum_{i=1}^{n} x_{i}\right) \geq \frac{1}{n} \sum_{i=1}^{n} f\left(x_{i}\right) \text { and } g\left(\frac{1}{n} \sum_{i=1}^{n} x_{i}\right) \leq \frac{1}{n} \sum_{i=1}^{n} g\left(x_{i}\right) .
$$


Since $f$ and $g$ are strictly increasing, also $f^{-1}$ and $g^{-1}$ are strictly increasing. Therefore, from (13) we obtain

$$
f^{-1}\left(\frac{1}{n} \sum_{i=1}^{n} f\left(x_{i}\right)\right) \leq f^{-1}\left(f\left(\frac{1}{n} \sum_{i=1}^{n} x_{i}\right)\right)=\frac{1}{n} \sum_{i=1}^{n} x_{i}=g^{-1}\left(g\left(\frac{1}{n} \sum_{i=1}^{n} x_{i}\right)\right) \leq g^{-1}\left(\frac{1}{n} \sum_{i=1}^{n} g\left(x_{i}\right)\right),
$$

which gives (12) and finishes the proof.

Example 5 Let us take $f(x)=\ln x$ and $g(x)=x^{2}, x>0$. Clearly, $f$ and $g$ satisfy the assumptions of Theorem 6. Since $f$ generates the geometric mean and $g$ generates the quadratic mean, from (12) we get

$$
G\left(x_{1}, \ldots, x_{n}\right) \leq A\left(x_{1}, \ldots, x_{n}\right) \leq K\left(x_{1}, \ldots, x_{n}\right),
$$

for all $x_{1}, \ldots, x_{n}>0$.

Now, take $f(x)=-\frac{1}{x}$ and $g(x)=x^{2}, x>0$. Since $f$ generates the harmonic mean, by (12) we obtain

$$
H\left(x_{1}, \ldots, x_{n}\right) \leq A\left(x_{1}, \ldots, x_{n}\right) \leq K\left(x_{1}, \ldots, x_{n}\right),
$$

for all $x_{1}, \ldots, x_{n}>0$.

\section{Lagrangian-type set-valued means}

Now, assume that $f: I \rightarrow \mathbb{R}$ is a continuous function. By the classical integral mean value theorem, for each $x_{1}, x_{2} \in I, x_{1}<x_{2}$, there exists $\xi \in\left(x_{1}, x_{2}\right)$ such that

$$
f(\xi)=\frac{1}{x_{2}-x_{1}} \int_{x_{1}}^{x_{2}} f(x) d x .
$$

If, moreover, $f$ is strictly monotonic, then $f^{-1}(f(\xi))=\xi$, and hence the function $L_{f}$ : $I^{2} \rightarrow I$ defined by

$$
L_{f}\left(x_{1}, x_{2}\right)= \begin{cases}f^{-1}\left(\frac{1}{x_{2}-x_{1}} \int_{x_{1}}^{x_{2}} f(x) d x\right) & \text { if } x_{1} \neq x_{2}, \\ x_{1} & \text { if } x_{1}=x_{2}\end{cases}
$$

is a mean. It is called the Lagrangian mean.

For a given set-valued map $F: I \rightarrow S(\mathbb{R})$ the integral of $F$ is understood in the sense of Aumann, i.e. it is the set of integrals of all Lebesgue integrable selections of this map (cf. [2]):

$\int_{a}^{b} F(x) d x=\left\{\int_{a}^{b} f(x) d x: f:[a, b] \rightarrow \mathbb{R}\right.$ is integrable and $\left.f(x) \in F(x), x \in[a, b]\right\}$.

Analogously as above we can define a potential set-valued Lagrangian-type mean for a map $F: I \rightarrow S(\mathbb{R})$ putting for $x_{1}, x_{2} \in I$

$$
L_{F}\left(x_{1}, x_{2}\right)= \begin{cases}F^{+}\left(\frac{1}{x_{2}-x_{1}} \int_{x_{1}}^{x_{2}} F(x) d x\right) & \text { if } x_{1} \neq x_{2}, \\ \left\{x_{1}\right\} & \text { if } x_{1}=x_{2}\end{cases}
$$

The next theorem gives conditions under which the map $L_{F}$ is a set-valued mean.

Theorem 7 Let $f, g: I \rightarrow \mathbb{R}$ be strictly increasing functions such that $f$ is concave and $g$ is convex. Assume that $f \leq g$ on $I$ and $F(x)=[f(x), g(x)]$ for all $x \in I$. Then the map $L_{F}: I^{2} \rightarrow S(I)$ given by (15) is a set-valued mean. 
Proof Note first that if $F(x)=[f(x), g(x)]$ with integrable $f, g: I \rightarrow \mathbb{R}$, then for each $x_{1}, x_{2} \in I, x_{1}<x_{2}$

$$
\int_{x_{1}}^{x_{2}} F(x) d x=\left[\int_{x_{1}}^{x_{2}} f(x) d x, \int_{x_{1}}^{x_{2}} g(x) d x\right],
$$

because $f, g$ are selections of $F$ and the Aumann integral is a convex set. Assume that $x_{1}, x_{2} \in I, x_{1}<x_{2}$ and fix $x \in L_{F}\left(x_{1}, x_{2}\right)$. Then

$$
F(x) \subset \frac{1}{x_{2}-x_{1}} \int_{x_{1}}^{x_{2}} F(x) d x=\frac{1}{x_{2}-x_{1}}\left[\int_{x_{1}}^{x_{2}} f(x) d x, \int_{x_{1}}^{x_{2}} g(x) d x\right],
$$

and hence

$$
f(x) \geq \frac{1}{x_{2}-x_{1}} \int_{x_{1}}^{x_{2}} f(x) d x \text { and } g(x) \leq \frac{1}{x_{2}-x_{1}} \int_{x_{1}}^{x_{2}} g(x) d x .
$$

By the integral mean value theorem there exist $\xi_{1}, \xi_{2} \in\left(x_{1}, x_{2}\right)$ such that

$$
f\left(\xi_{1}\right)=\frac{1}{x_{2}-x_{1}} \int_{x_{1}}^{x_{2}} f(x) d x \text { and } g\left(\xi_{2}\right)=\frac{1}{x_{2}-x_{1}} \int_{x_{1}}^{x_{2}} g(x) d x .
$$

Since $f$ and $g$ are strictly increasing, by (16) and (17) we get

$$
x \geq \xi_{1}>x_{1} \text { and } x \leq \xi_{2}<x_{2},
$$

which proves that $L_{F}\left(x_{1}, x_{2}\right) \subset \operatorname{conv}\left\{x_{1}, x_{2}\right\}$.

Now, we will prove that $L_{F}\left(x_{1}, x_{2}\right)$ is nonempty. Since $f$ is concave and $g$ is convex, by the Hermite-Hadamard theorem we have

$$
f\left(\frac{x_{1}+x_{2}}{2}\right) \geq \frac{1}{x_{2}-x_{1}} \int_{x_{1}}^{x_{2}} f(x) d x \text { and } g\left(\frac{x_{1}+x_{2}}{2}\right) \leq \frac{1}{x_{2}-x_{1}} \int_{x_{1}}^{x_{2}} g(x) d x .
$$

From here

$$
F\left(\frac{x_{1}+x_{2}}{2}\right)=\left[f\left(\frac{x_{1}+x_{2}}{2}\right), g\left(\frac{x_{1}+x_{2}}{2}\right)\right] \subset \frac{1}{x_{2}-x_{1}}\left[\int_{x_{1}}^{x_{2}} f(x) d x, \int_{x_{1}}^{x_{2}} g(x) d x\right] .
$$

Consequently,

$$
\frac{x_{1}+x_{2}}{2} \in F^{+}\left(\frac{1}{x_{2}-x_{1}} \int_{x_{1}}^{x_{2}} F(x) d x\right)=L_{F}\left(x_{1}, x_{2}\right) .
$$

This shows that $L_{F}\left(x_{1}, x_{2}\right) \neq \emptyset$ and finishes the proof.

Acknowledgements The author express his gratitude to the anonymous referees for their careful reading and detailed comments which have improved the paper.

Open Access This article is licensed under a Creative Commons Attribution 4.0 International License, which permits use, sharing, adaptation, distribution and reproduction in any medium or format, as long as you give appropriate credit to the original author(s) and the source, provide a link to the Creative Commons licence, and indicate if changes were made. The images or other third party material in this article are included in the article's Creative Commons licence, unless indicated otherwise in a credit line to the material. If material is not included in the article's Creative Commons licence and your intended use is not permitted by statutory regulation or exceeds the permitted use, you will need to obtain permission directly from the copyright holder. To view a copy of this licence, visit http://creativecommons.org/licenses/by/4.0/. 


\section{References}

1. Aczél, J., Dhombres, J.: Functional Equations in Several Variables with Applications to Mathematics, Information Theory and to the Natural and Social Sciences. Cambridge University Press, Cambridge (1989)

2. Aubin, J.-P., Frankowska, H.: Set-valued Analysis. Birkhäuser, Boston-Basel-Berlin (1990)

3. Beckenbach, B., Bellman, R.: An introduction to inequalities. Random House and Yale University (1961)

4. Bullen, P.S.: Handbook of Means and Their Inequalities. Kluwer, Dordrecht (2003)

5. Cauchy, A.L.: Cours D'analyse De L'École Royale Polytechnique, $I^{r e}$ Partie: Analyse Algébrique. Imprimérie Royale, Paris (1821)

6. Daróczy, Z., Páles, Z.: Gauss-composition of means and the solution of matkowski-sutô problem. Publ. Math. Debrecen 61, 157-218 (2002)

7. Elezović, N.: Asymptotic inequalities and comparison of classical means. J. Math. Ineq. 9, 177-196 (2015)

8. Jarczyk, J., Jarczyk, W.: Invariance of means. Aequat. Math. 92, 801-872 (2018)

9. Makó, J., Páles, Z.: The invariance of the atithmetic mean with respect to generalized quasi-arithmetic means. J. Math. Anal. Appl. 353, 8-23 (2009)

10. Matkowski, J.: Generalized weighted arithmetic means. In: Rassias, T., Brzdȩk, J. (eds.) Functional Equations in Mathematical Analysis, pp. 563-582. Springer, New York (2012)

11. Nikodem, K.: $K$-convex and $K$-concave set-valued functions, Zeszyty Nauk. Politech. Łódz. Mat. 559(Rozprawy Nauk 114 Łódź), 1-75 (1989)

12. Toader, G., Costin, I.: Means in Mathematical Analysis. Bivariate Means. Mathematical Analysis and Its Applicatio Series. Academic Press, An imprint of Elsevier, London (2018)

Publisher's Note Springer Nature remains neutral with regard to jurisdictional claims in published maps and institutional affiliations. 